\title{
Riscos psicossociais em unidades especializadas: implicações para a formação e a saúde do enfermeiro residente
}

\author{
Psychosocial risks in specialized units: implications for the training and health of \\ nurse residents
}

\author{
Riesgos psicosociales en unidades especializadas: repercusiones para la formación y la \\ salud del enfermero residente
}

\author{
Luana dos Santos Vasconcellos Lima'; Elias Barbosa de Oliveira'; Maria Yvone Chaves Mauro III. \\ Márcia Tereza Luz Lisboa ${ }^{I V}$; Luciana Guimarães Assadv; Renata dos Anjos Correa Carvalho ${ }^{V I}$
}

\begin{abstract}
RESUMO: Objetivou-se neste estudo identificar os fatores de risco psicossocial presentes em unidades especializadas, na visão do enfermeiro residente e analisar como os mesmos afetam a saúde do grupo. Método qualitativo, descritivo, tendo como campo cinco unidades especializadas de um hospital universitário, situado no município do Rio de Janeiro (Brasil). Utilizou-se a técnica de entrevista semiestruturada com 20 residentes, em 2012. Aplicada a análise de conteúdo aos depoimentos, chegou-se aos seguintes resultados: na visão do enfermeiro residente, os riscos psicossociais acarretam prejuízos ao processo de formação e estresse ocupacional, sendo alguns deles: recursos humanos e materiais insuficientes, conflitos no trabalho em equipe e a pouca autonomia. Concluiu-se que há necessidade de as instituições envolvidas no processo de formação promoverem o gerenciamento dos riscos presentes em unidades especializadas, de modo a minimizar os prejuízos acarretados ao processo de formação e à saúde do grupo. Palavras-Chave: Enfermagem; estresse; controle de riscos; saúde mental.
\end{abstract}

\begin{abstract}
This study aimed to identify psychosocial risks in specialized units in the view of nursing residents, and to examine how psychosocial risks affect this group's health. A qualitative and descriptive method was applied at five specialized units at a university hospital in Rio de Janeiro City, Brazil. Semi-structured interviews of 20 nursing residents were conducted in 2012. Content analysis led to the following results: in the nursing residents' view, psychosocial risks hinder the training process and cause occupational stress. They include: insufficiency of human and material resources, conflicts within work teams, and lack of autonomy. It was concluded that the institutions involved in the training process must engage in risk management at specialized units, so as to minimize harm to the training process and the group's health.

Keywords: Nursing; stress; risk control; mental health.
\end{abstract}

RESUMEN: Se objetivó en este estudio identificar los factores de riesgo psicosocial presentes en unidades especializadas, en la visión del enfermero residente y analizar cómo influyen en la salud del grupo. Método cualitativo, descriptivo, teniendo como campo cinco unidades especializadas de un hospital universitario, situado en el municipio de Rio de Janeiro (Brasil). Se utilizó la técnica de entrevista semiestructurada con 20 enfermeros residentes, en 2012. Se aplicó a las declaraciones el análisis de contenido y se llegó a los siguientes resultados: en la visión del enfermero residente, los riesgos psicosociales acarrean perjuicios al proceso de formación y estrés laboral, siendo algunos de ellos: recursos materiales y humanos insuficientes, conflictos en el trabajo en equipo y la restricta autonomía. Se llegó a la conclusión de que hace falta que las instituciones involucradas en el proceso de formación promuevan la gerencia de los riesgos en unidades especializadas, de modo a minimizar los perjuicios acarreados al proceso de formación y a la salud del grupo.

Palabras Clave: Enfermería; estrés; control de riesgos; salud mental.

\section{INTRODUÇÃO}

Instituições da área da saúde, principalmente os hospitais, que possuem programas de residência em enfermagem e em outras áreas, têm grande respon- sabilidade quanto a alocação de recursos humanos e materiais, assim como estabelecer uma estrutura que possibilite a capacitação de pessoal para o Sistema

\footnotetext{
IMestre em Enfermagem. Especialista em Enfermagem do Trabalho. Enfermeira do Hospital Central da Polícia Militar do Estado do Rio de Janeiro. Brasil. E-mail: lulusvl@gmail.com

IIPós Doutor em Alcool e Drogas. Doutor em Enfermagem. Professor Adjunto de Pós-Graduação (Mestrado) e Graduação da Faculdade de Enfermagem da Universidade do Estado do Rio de Janeiro. Líder do Grupo de Estudos Saúde Mental e Trabalho. Rio de Janeiro, Brasil. E-mail: eliasbo@oi.com.br

IIIDoutora em Enfermagem. Professora Titular Visitante da Universidade do Estado do Rio de Janeiro. Presidente da Associação Nacional de Enfermagem do Trabalho - Seção Rio de Janeiro. Brasil. E-mail: mycmauro@uol.com.br

IV Doutora em Enfermagem. Professora Associada do Departamento de Enfermagem Fundamental da Escola de Enfermagem Anna Nery da Universidade Federal do Rio de Janeiro. Membro do Núcleo de Pesquisa Enfermagem e Saúde do Trabalhador e Núcleo de Pesquisa de Fundamentos do Cuidado de Enfermagem. Rio de Janeiro, Brasil. E-mail: marcialuzlisboa@gmail.com

${ }^{v}$ Doutora em Enfermagem. Professora Adjunta e Coordenadora da Especialização (Modalidade Residência) em Enfermagem da Faculdade de Enfermagem da Universidade do Estado do Rio de Janeiro. Brasil. E-mail: lgassad@gmail.com

${ }^{\mathrm{V}}$ Mestre em Enfermagem. Especialista em Enfermagem em Nefrologia (Residência). Enfermeira da Clinica de Doenças Renais. Rio de Janeiro, Brasil.

E-mail: E-mail: enfaredacorrea@gmail.com
} 
Único de Saúde (SUS) e o desenvolvimento de práticas seguras nos níveis técnico, assistencial e gerencial ${ }^{1}$.

Questões relacionadas ao processo saúde doença e aos riscos envolvidos na formação, também devem ser contempladas na política de recursos humanos das instituições, cabendo ao serviço de saúde ocupacional, em parceria com o serviço de educação continuada e instituições de ensino, papel relevante acerca da promoção de condições dignas de trabalho e formação, no intuito de promover a capacitação, a saúde e o bem estar desta parcela de trabalhadores.

Para ingressar no mundo do trabalho e atender as exigências impostas pelo mesmo, muitos enfermeiros optam por se especializarem nos moldes de residência. Esta modalidade de ensino visa garantir segurança no desempenho e desenvolvimento de habilidades teóricas e práticas, proporcionando crescimento profissional e entrada em um mercado de trabalho cada vez mais competitivo e exigente ${ }^{2}$.

Desse modo, o residente, ao optar pela especialização na modalidade de residência em unidades hospitalares complexas e diante de condições inadequadas de trabalho, se expõe a riscos ocupacionais e entre eles, os psicossociais. Tais riscos respondem pelo estresse ocupacional, que mantém relação estreita com as exigências físicas e psicológicas do profissional, atrelados às jornadas extensas, às questões de relacionamento interpessoal e às exigências para o cuidado de pacientes com risco de complicação e morte ${ }^{3}$.

Alerta-se para o fato de que residentes de enfermagem por serem, em sua maioria, recém-formados e inexperientes, possuem estratégias reduzidas de enfrentamento das situações geradores de desgaste, principalmente em ambientes de trabalho precários e com déficit de pessoal. $O$ desgaste é acentuado nas situações onde o especializando é contabilizado como parte da equipe, realizando atividades nem sempre inerentes a sua função, o que é uma contradição ao que se propõem os programas de residência ${ }^{4}$.

Em estudo realizado com residentes de enfermagem, identificou-se que a carga horária extensa, o trabalho em turnos, os conflitos no relacionamento interpessoal e demais exigências da organização hospitalar e do próprio processo de formação afetavam de modo substancial a saúde do especializando. Entre os principais achados, evidenciaram-se problemas como: sintomas depressivos, psicossomáticos, retração social e inibição para o trabalho, interferindo na qualidade de vida e no trabalho 5 .

Considerando a relevância de programas institucionais voltados para o manejo do estresse ocupacional em enfermeiros residentes e a necessidade de contribuir para o desenvolvimento da área, o presente estudo teve como objetivos identificar os fatores de risco psicossocial presentes em unidades especializa- das, na visão do enfermeiro residente, e analisar como os mesmos afetam a saúde do grupo estudado.

\section{REVISÃo DE Literatura}

De acordo com o artigo $3^{\circ}$ da Resolução ${ }^{\circ}$ 2/2012, da Secretaria de Educação Superior do Ministério de Educação (MEC), a residência em enfermagem é uma modalidade de ensino de pós-graduação latu senso, sob a forma de curso de especialização, caracterizado por ensino em serviço, com carga horária de 60 horas semanais e duração mínima de 2 anos $^{6}$. Possui como base a aprendizagem pela prática cotidiana, marcada pela aquisição progressiva de atributos técnicos e relacionais de suma relevância no desenvolvimento do futuro profissional? .

No entanto, esta modalidade de ensino ao inserir na formação atividades de cunho prático que, indubitavelmente contribui para o processo de construção de competências e habilidades de cunho técnico e relacional, pode expor o residente a riscos ocupacionais. Tais riscos afetam a saúde e bem estar do grupo, principalmente ao se considerar o despreparo, a pouca experiência na profissão e a inexistência de vínculo empregatício na instituição $0^{4}$.

Dentre os riscos que afetam a saúde do residente, existem os psicossociais, que se relacionam ao planejamento, a organização, o gerenciamento do trabalho e o contexto ambiental e social, os quais apresentam potencial de acarretar prejuízos físico, social e psicológico ao grupo. Portanto, deve-se realizar uma adequada avaliação dos riscos no trabalho da enfermagem para preveni-los, elimina-los ou minimiza-los, o que possibilita a redução de problemas como queda da produtividade, custos sociais e econômicos decorrentes do adoecimento e absenteísmo por causas diversas ${ }^{8}$.

No ambiente de trabalho hospitalar os riscos psicossociais acarretam estresse ocupacional com implicações para a saúde do indivíduo e a qualidade do serviço ofertado. Na gênese do estresse e sua correlação com os fatores de risco psicossocial deve-se considerar: inexistência de programas voltados para o preparo e/ou capacitação do trabalhador, inadequação das condições de trabalho, ambiguidade de papéis, longas jornadas de trabalho, relacionamento interpessoal conflituoso e a difícil conciliação entre o trabalho e as atividades de cunho social e familiar?.

Os estudos sobre os riscos psicossociais são recentes e apoiados na epidemiologia social. Entretanto, ao utilizar critérios somáticos e biológicos na avaliação e privilegiar a quantificação, esta vertente não se aproxima dos elementos inerentes à significação nem à vivência subjetiva dos trabalhadores. Este papel tem cabido à psicodinâmica do trabalho ao enfatizar a centralidade do trabalho na vida dos sujeitos, analisando os aspectos inerentes desta atividade que podem 
favorecer a saúde ou a doença com possibilidades de investigação e intervenção nos locais de trabalho ${ }^{10}$.

Portanto, por compreender a residência como um curso de especialização que objetiva inserir o profissional no mundo do trabalho, as instituições, que desenvolvem esta modalidade de ensino, devem ter como princípio, as ações e diretrizes assentadas na política de saúde do trabalhador para o SUS com vistas à promoção da saúde e a redução da morbimortalidade deste grupo de trabalhadores ${ }^{1}$.

\section{Metodologia}

Estudo qualitativo e descritivo que originou do desejo de conhecer os fatos observados, registrando, analisando, classificando e interpretando-os no intuito de descrever os fenômenos da realidade ${ }^{11}$. $O$ campo foi um hospital universitário de grande porte, situado no município do Rio de Janeiro, considerado centro de referência nas áreas de ensino de graduação e de aperfeiçoamento dos conhecimentos para graduados e pós-graduados, através de programas de especialização em várias áreas.

Participaram do estudo 20 residentes de enfermagem, matriculados nos programas de terapia intensiva, cirurgia cardíaca, neonatologia e clínica médica. Os critérios adotados na inclusão foram: residentes do primeiro e segundo ano, frequentando regularmente os respectivos programas e que, na ocasião em que os dados foram coletados, estivessem lotados em unidades especializadas. Excluídos os residentes que se encontravam de férias e/ou afastados do programa devido a problemas de saúde e licenças de outra natureza.

Realizados o convite, as explanações acerca da pesquisa e o agendamento para as entrevistas, os dados foram coletados individualmente, na própria unidade, onde o residente realizava as suas atividades. Em atenção à Resolução n ${ }^{\circ}$ 466/12, o entrevistado se certificou da autorização do estudo pelo Comitê de Ética em Pesquisa (protocolado sob o $\mathrm{n}^{\circ}$ 067/2012) e assinou o Termo de Consentimento Livre e Esclarecido.

Informou-se que a participação dos residentes seria voluntária e que teriam o direito de se retirar da pesquisa em qualquer fase. Garantiu-se o sigilo dos dados e ratificou-se que os resultados seriam apresentados em eventos e publicados em revistas científicas. Na obtenção dos depoimentos, utilizou-se a técnica de entrevista semiestruturada ${ }^{11}$, mediante um roteiro que combinou perguntas fechadas e abertas, possibilitando ao residente discorrer sobre o processo de formação, as condições de trabalho e os riscos psicossociais. As respostas foram gravadas em meio digital e com o intuito de preservar o anonimato, na transcrição dos depoimentos, foram utilizadas as letras Rd em ordem numérica, de acordo com a entrada no texto.

Realizada a transcrição, a linguagem foi analisada mediante a técnica de análise de conteúdo do tipo temática, que se baseou na decodificação do texto em diversos elementos, os quais foram classificados e formaram agrupamentos analógicos ${ }^{12}$, em que chegou-se às seguintes categorias: a atividade assistencial e gerencial do enfermeiro residente; a reduzida autonomia do enfermeiro residente frente à equipe; e relacionamento interpessoal conflituoso.

\section{Resultados e Discussão}

\section{A atividade assistencial e gerencial do enfer- meiro residente}

De acordo com o programa de residência, cabe ao enfermeiro residente o cuidado integral ao paciente crítico. Afinal, são pacientes instáveis, portanto sujeitos a complicações, necessitando de profissionais altamente capacitados e familiarizados com o processo de trabalho. Desse modo, os residentes se reportaram ao trabalho em unidades especializadas como uma atividade que exige esforço físico, mental e realização de intervenções contínuas, devido à imprevisibilidade do quadro clínico dos pacientes.

Geralmente o residente fica com o paciente mais grave! [...] é assistência mesmo! Banho no leito, administração de medicamentos, plano de cuidados! Então você fica, assim, gerenciando o cuidado total desse paciente. (Rd2)

O paciente da cardíaca é um paciente naturalmente grave! Mesmo que você diga: 'Ah! Está em um pósoperatório tranquilo!' Mas sempre tem o risco de ter uma parada cardíaca. (Rd6)

É um trabalho que exige muita força! Muita atenção! Memória! Porque a gente lida todo o momento com pacientes graves, que podem se instabilizar! (Rd10)

As unidades críticas no hospital geral são setores com maiores demandas físicas e psicológicas, concentrando recursos tecnológicos de ponta e pessoal altamente especializado. Esses setores, por suas características, são considerados como os mais agressivos, tensos e traumatizantes pelo tipo de clientela e por exigir do profissional conhecimentos específicos e capacidade de agir diante de situações imprevistas ${ }^{13}$.

Além da assistência ao paciente crítico que implica desgaste, outro aspecto apontado pelo grupo foi a incorporação de tecnologias duras ao trabalho e as dificuldades na sua utilização. Como relatado, durante a graduação, nem sempre o aluno tem oportunidade de desenvolver as habilidades em termos de domínio tecnológico, sendo a residência uma estratégia de ensino teórico-prático que possibilita, ao especializando, suprir esta lacuna.

Na graduação, eu não tive tanto contato com essa tecnologia mais específica. Então, você tem aquele choque inicial e depois você vai se acostumando, por estar todo dia ali praticando e tendo contato com a tecnologia. (Rd12) 
A questão dos aparelhos, no inicio é um pouco mais difícil! Você não está acostumado! Depois a bomba infusora é mais fácil de você mexer. Acho que o respirador é a tecnologia que dá mais medo. (Rd17)

A tecnologia é uma coisa que estressa muito o residente [... várias vezes eu sai da terapia intensiva e, em casa, tomando banho, escutava o ventilador mecânico. (Rd13)

Identificou-se que a incorporação da tecnologia dura em unidades especializadas, na visão do residente, é um fator de risco psicossocial por causar insegurança, estresse e exigir do grupo habilidades e conhecimentos específicos em seu manuseio. Salienta-se a importância da supervisão e condução do especializando por preceptores, considerados profissionais mais experientes, de modo a proporcionar maior segurança no desempenho e minimizar a possibilidade de erros e iatrogenias.

$\bigcirc$ enfermeiro residente de unidades especializadas é susceptível ao estresse ocupacional, sendo o desgaste acentuado, em função dos esforços despendidos no cuidado de pacientes críticos, que exigem observação e controle extenuantes. Deve-se considerar, na gênese do desgaste, a inexperiência e a falta de familiaridade com o aparato tecnológico em uso; portanto, um trabalho que se caracteriza pelo alto grau de responsabilidade ${ }^{3}$.

Quanto à incorporação das tecnologias duras ao trabalho da enfermagem, salienta-se a importância de uma política voltada para a prevenção de riscos para o paciente e a equipe, através da manutenção preventiva e corretiva de aparelhos em uso, treinamento das equipes, condições adequadas de trabalho e suporte de serviços especializados. Desse modo, minimiza-se a possibilidade de erros e iatrogenias, como também o envolvimento do profissional em questões éticas e legais relacionadas ao exercício profissional ${ }^{14}$.

No entendimento dos participantes, o residente do segundo ano, por conhecer mais de perto os problemas relativos à unidade e ter maior domínio sobre o processo de trabalho, acaba assumindo outras atividades de responsabilidade do preceptor, entre elas: o treinamento de pessoal de nível técnico, o recebimento do plantão e a gerencia da unidade. Em outros momentos, como referido pelo grupo, as atividades são compartilhadas com os demais profissionais.

Então a gente fica responsável por tudo! Fica responsável pelo treinamento de funcionários! Responsável por assumir o plantão! E tudo o que acontece no plantão é responsabilidade do residente. (Rd4)

Nós, como residentes, assumimos tanto a parte assistencial quanto a gerencial! Quando há enfermeiro plantonista, a gente divide! Quando não há, a gente assume tudo. E é uma assistência completa. (Rd7)

É o residente que esta ali todos os dias! Que conhece o paciente diariamente! Sabe todos os casos! Sabe todas as histórias! Conhece, desde o inicio, o paciente! (Rd6)
Tais evidências devem ser analisadas com cautela, pois apesar de a residência oferecer oportunidade de crescimento, liderança e desenvolvimento de habilidades relacionadas ao cuidado do paciente crítico, a presença do preceptor é essencial em termos de supervisão e suporte de práticas seguras. Cabe à instituição formadora prover as unidades de profissionais capacitados para a função, evitando que o residente assuma funções para as quais ainda não se encontra preparado. Infere-se que as atividades de cunho gerencial e assistencial acumuladas podem sobrecarregar o especializando e, quando acrescidas, concomitantemente, à função de ensino - aprendizagem, podem repercutir de forma negativa na saúde, na formação e na qualidade do serviço prestado.

Para que o programa de Residência em Enfermagem seja legalmente reconhecido, necessita de corpo docente e preceptores especializados. $\mathrm{O}$ enfermeiro preceptor é corresponsável pela supervisão, orientação e suporte do residente que se inspira no profissional para aprimorar as suas habilidades. Portanto, a ausência do preceptor pode contribuir para sentimentos de frustração vivenciados pelo residente, que diante de determinadas circunstancias acaba por assumir a responsabilidade pelo próprio desenvolvimento de suas habilidades ${ }^{15}$.

\section{A reduzida autonomia do enfermeiro residente frente à equipe}

A transição do meio acadêmico para o trabalho pode se constituir em um fator de risco psicossocial com repercussões para a saúde mental do recémformado, diante das exigências da organização e os recursos internos que o indivíduo possui para o enfrentamento das situações de conflito e/ou urgências. Como relatado, os residentes, principalmente do primeiro ano, por serem inexperientes e não dominarem o processo de trabalho possuem pouca autonomia no trabalho, sendo esta problemática agravada pelo fato de não terem o seu papel como enfermeiro reconhecido pela equipe.

Inicialmente você chega e não tem autonomia nenhuma. Você nem é encarado como enfermeiro. As pessoas ainda acham que você é acadêmico de enfermagem. Então você não tem autonomia nenhuma. (Rd14)

No primeiro ano é muito pouca a autonomia! Eu até entendo! É um processo natural mesmo, pois você chega, muitas vezes, sem experiência, sem prática. (Rd20)

Essa autonomia de dizer o que deve ou não fazer para a equipe! Isso enquanto R1 eu não vejo não. Eu vejo mais com o R2. (Rd11)

A autonomia laboral é uma categoria que remete a escolhas do que fazer e como fazer o trabalho. Em situações laborais onde existe reduzida autonomia, o estresse tende a se intensificar devido às pressões decorrentes de cobranças, do cumprimento de metas 
e prontidão para a resolução de problemas. Quanto à residência em si, existem normas estabelecidas no programa em termos de carga horária a ser cumprida, atividades de cunho acadêmico e realização de plantões de acordo com a necessidade do serviço, devendo o residente se adaptar as exigências da organização ${ }^{3}$.

Ratifica-se que a autonomia só é possível para o enfermeiro quando este se concentra em atividades que ele realmente domina, sendo capaz de agir frente às situações não previstas. Desse modo, a autonomia é possível na medida em que o profissional, ao longo do seu exercício profissional, desenvolve competências e habilidades nos campos técnico, assistencial e relacional ${ }^{16}$.

O enfermeiro residente, por não fazer parte do efetivo de trabalhadores e ter reduzida autonomia em termos de poder decisório, enfrenta situações conflitantes em relação ao desempenho da equipe de enfermagem, o que gera tensão e angústia. Desse modo, os depoimentos perpassaram a necessidade sentida pelo especializando do reconhecimento do seu papel pela equipe de enfermagem, faltando por parte do efetivo de trabalhadores, clareza de sua função e responsabilidades no contexto do trabalho.

Especificamente o problema que eu passei está mais em relação à equipe mesmo! Porque tem momentos em que somos cobrados por sermos residentes! Ora não somos respaldados em cobrar da equipe as coisas, por não fazermos parte, teoricamente, da equipe. Sermos apenas alunos estagiando. (Rd11)

Você não tem autonomia para fazer as coisas! Você só vai ter essa autonomia quando você estiver num plantão que não tenha preceptor, não tenha enfermeiro! (Rd12)

A falta de clareza, por parte da equipe, quanto às funções e às responsabilidades do enfermeiro residente na estrutura organizacional, reflete a fragilidade do processo de capacitação e a percepção de reduzida autonomia relatadas pelo especializando. Cabe, portanto, à preceptoria ações concretas no sentido de trabalhar, junto à equipe, o papel desempenhado pelo residente, o que pode minimizar os conflitos e oportunizar a realização de um trabalho coletivo. Infere-se que o residente, ao optar pela especialização, possui expectativas de aprimoramento e crescimento profissional que, se não forem alcançados, poderão levar à desmotivação e até mesmo à evasão do programa.

Portanto, pensar no hospital como unidade de cuidados e de formação profissional é apontar para a organização desse sistema, considerando a importância dos aspectos subjetivos envolvidos e entre eles: a necessidade de autonomia do especializando, o reconhecimento do trabalho realizado, as relações pautadas no respeito às diferenças e atitudes de acolhimento por parte dos demais membros das equipes - aspectos fundamentais e que vão influenciar a atuação do enfermeiro residente nos diferentes espaços da saúde ${ }^{16}$.

\section{Relacionamento interpessoal conflituoso}

O bom relacionamento interpessoal é essencial na condução do trabalho em equipe, podendo ser um fator de realização e satisfação. Em caso negativo leva a situações de conflitos e disputas, envolvendo várias categorias profissionais, devido às diferenças de formação e visões de mundo. Os residentes referiram o relacionamento interpessoal conflituoso no cotidiano laboral, envolvendo membros da equipe de enfermagem e preceptores.

Eu tive problemas de relacionamento pessoal com a equipe! Na verdade alguns profissionais parecem ter alguma resistência com os residentes. (Rd10)

O problema que eu passei é mais em relação à equipe mesmo [...] e muitas vezes a falta de respeito com os residentes. (Rd11)

O maior problema durante a residência é a relação interpessoal complicada, principalmente com os preceptores. (Rd16)

Entre as situações vivenciadas no cotidiano do trabalho da enfermagem, o relacionamento interpessoal é apontado como o principal indutor de estresse nesses profissionais, em decorrência de fatores como: a falta de poder e influência, incompatibilidade na interação com superior hierárquico, subordinados pouco competentes e o fato de os enfermeiros se sentirem sós frente à necessidade de tomada de decisões ${ }^{17}$. Uma problemática que pode ocorrer nos programas de residência em enfermagem é o conflito entre residente e preceptor, quando enfermeiros delegam as suas atividades para o residente sem fornecer as orientações técnicas dos procedimentos ou os encaminhamentos relacionados a estratégias de aprendizagem ${ }^{6}$.

\section{Conclusão}

O estudo evidenciou que o enfermeiro, ao optar pela residência em unidades especializadas, está exposto a fatores de risco psicossociais relacionados à estrutura e a organização do trabalho que afetam o seu bem-estar e saúde. No que diz respeito à atividade gerencial, o residente, principalmente do segundo ano, diante das pressões sociais do trabalho, é contado como parte da equipe, substituindo o trabalho do preceptor e, em algumas situações, do técnico de enfermagem.

Esta situação de trabalho/aprendizagem, apesar de ser um desafio para a prática profissional e contribuir para a sua formação em termos de aquisição de habilidades, liderança da equipe e demais competências, deve ser analisada com cautela, pois a presença do preceptor é indispensável em termos de orientação, suporte do grupo e supervisão de práticas seguras.

No que diz respeito ao cuidado de pacientes críticos e todo arsenal tecnológico utilizado, cabe à instituição formadora prover o serviço de pessoal e infraes- 
trutura adequados, de modo a minimizar o desgaste do especializando diante do alto grau de responsabilidade envolvido na assistência ao paciente crítico.

Quanto à reduzida autonomia do residente frente à equipe, trata-se de uma questão dialética, na medida em que o próprio residente, principalmente do primeiro ano, reconhece que o trabalho, em unidades especializadas, possui demandas de cunho técnico e assistencial que extrapolam seus recursos e para as quais não se sente preparado, fazendo, portanto parte do processo de formação.

Sobre o relacionamento interpessoal, identificou-se que o mesmo, por vezes, torna-se conflituoso pela falta de clareza da equipe em relação ao papel do residente, que apesar de em algumas ocasiões assumir a liderança do grupo, em outras situações tem de se submeter à realização de atividades de cunho técnico assistencial sem a devida orientação.

Apesar das limitações do estudo, decorrentes do reduzido número de participantes e de único cenário amostra e da impossibilidade de generalizações para outros contextos de formação, ratifica-se a sua relevância ao identificar a influência dos fatores psicossociais do trabalho para a formação, a satisfação e a saúde dos residentes. Pelo fato de a residência ser uma modalidade de ensino teórico e prático relevante na área de capacitação de recursos humanos, confirmase o papel das instituições envolvidas nesse processo ao promover o gerenciamento dos riscos, apontados pelo residente, de modo a minimizar os prejuízos acarretados ao processo de formação e a saúde do grupo.

\section{REFERÊNCIAS}

1.Aguiar BGC. O que é a residência de enfermagem. In: Ministério da Saúde (Br). Guia de orientações para o enfermeiro residente. Brasília (DF), 2005. [citado em 10 set 2014] Disponível em: http://biblioteca.claretiano. edu.br/phl8/pdf/05_0004_M.pdf

2.Santos VP, Whitaker IY, Zanei SSV. Especialização em Enfermagem Modalidade Residência em Unidade de Terapia Intensiva: egressos no mercado de trabalho. Rev Gaúcha Enferm [internet] 2007 [citado em 14 out 2014] 28:193-9. Disponível em: http://seer.ufrgs.br/index.php/ RevistaGauchadeEnfermagem/article/view/3163/1734 3.Oliveira EB, Souza NVM, Chagas SCS, Lima LSV, Correa RA . Esforço e recompensa no trabalho do enfermeiro residente em unidades especializadas. Rev enferm UERJ [internet] 2013 [citado em 9 set 2014] 21: 173-8. Disponível em: http://www.facenf.uerj.br/v21n2/v21n2a06.pdf 4.Pinheiro J, Zeitoune RCG. O trabalho dos residentes de enfermagem na perspectiva dos riscos ocupacionais. HU Revista, Juiz de Fora [internet] 2011 [citado em 07 ago 2014] 37: 225-32. Disponível em: http://www.seer. ufjf.br/index.php/hurevista/article/viewFile/1430/547

5.Franco GP, Barros ALBL, Martins LAN. Burnout em residentes de enfermagem. Rev esc enferm USP. [internet] 2011 [citado 04 out 2014] 45:12-8. Disponível em: http:// www.scielo.br/scielo.php?script $=$ sci_arttext\&pid $=$ S0080 2342011000100002\&lng=e\&nrm =iso\&tlng=enhttp:// www.scielo.br/pdf/reeusp/v37n3/08.pdf

6. Ministério da Educação (Br). Comissão Nacional de Residência Multiprofissional em Saúde. Resolução nº 2 de 13 de abril de 2012. Dispõe sobre as Diretrizes Gerais para os Programas de Residência Multiprofissional e em Profissional de Saúde. Diário Oficial da União 2012 abr; n 73, Seção 1:24-5.

7. Barbeiro FMS, Miranda LV, Souza SR. Enfermeiro preceptor e residente de enfermagem: a interação no cenário da prática. R pesq Cuid Fundam Online [internet]. 2010 [citado em 07 ago 2014] 2:1080-7. Disponível em: http:// www.seer.unirio.br/index.php/cuidadofundamental/article/view/584/pdf_45

8.Camelo SHH, Angerami ELS. Riscos psicossociais no trabalho que podem levar ao estresse: uma analise da literatura. Cienc Cuid Saúde [internet]. 2008 [citado em 10 set 2014] 7: 232-40. Disponível em: http://periodicos.uem. br/ojs/index.php/CiencCuidSaude/article/view/5010/3246 9.Oliveira EB, Ferreira JMGF, Souza NVM, Costa HF, Nascimento LP. Produção do conhecimento da enfermagem sobre os riscos psicossociais no trabalho: revisão sistematizada da literatura. Rev Enfermagem Atual. 2010; 60: 31-4. 10.Fernandes SMBA, Medeiros SM, Ribeiro LM. Estresse ocupacional e o mundo do trabalho atual: repercussões na vida cotidiana das enfermeiras. Rev Eletr Enferm. [periódico na internet] 2008 [citado em 08 jul 2014];10:414-27.Disponível em: http://www.fen.ufg.br/ revista/v10/n2/v10n2a13.htm.

11.Polit DF, Beck CT, Hungler, BP. Fundamentos de pesquisa em enfermagem: métodos, avaliação e utilização. 5 ed. Porto Alegre (RS): Artmed; 2004.

12.Bardin, L. Análise de conteúdo. Lisboa (Pt): Edições 70; 2012 .

13.Coronetti, A, Nascimento ERP, Barra DCC, Martins JJ. O estresse da equipe de enfermagem na unidade de terapia intensiva: o enfermeiro como mediador. Revista Arq Catar Med [periódico na internet]. 2006 [citado em 04 out 2014] 36-43. Disponível em: http://www.acm.org. br/revista/pdf/artigos/394.pdf

14.Peres EF, Oliveira EB, Dantas NVS, Lisboa MTL, Silvino ZR. Segurança no desempenho e minimização de riscos em terapia intensiva. Rev enferm UERJ [internet]. 2013 [citado em 28 set 2014]. Disponível em: http:// www.facenf.uerj.br/v22n3/v22n3a06.pdf 15.Lyra ES, Pereira GL, Aguiar BGC. Preceptoria do enfermeiro residente: cooperação técnica. Anais do 61ํㅡㅁ gresso Brasileiro de Enfermagem: Transformação e sustentabilidade ambiental. Associação Brasileira de Enfermagem [internet] 2009 [citado 03 out 2014]. Disponível em: http:// www.abeneventos.com.br/anais_61cben/files/01970.pdf 16.Bueno FMG, Queiroz MS. O enfermeiro e a construção da autonomia profissional no processo de cuidar. Rev bras enferm [internet]. 2006 [citado em 02 jul 2014] 59: 222-7. Disponível em: http://www.scielo.br/pdf/reben/v59n2/a19.pdf 17.Montanholi LL, Tavares DMS, Oliveira GR. Estresse: fatores de risco no trabalho do enfermeiro hospitalar. Rev Bras Enferm [internet] 2006 [citado em 03 nov 2014] 59:5-13. Disponível: http://www.scielo.br/pdf/reben/ v59n5/v59n5a13.pdf 